\title{
A magnifying glass for the study of coupled developmental changes: Combining psychological networks and latent growth models
}

Running title: Psychological networks and latent growth models

\author{
Marie K. Deserno ${ }^{a 1}$ \\ Maien S.M. Sachisthal ${ }^{\mathrm{b}}$ \\ Sacha Epskamp bd \\ Maartje E.J. Raijmakers ${ }^{b c}$
}

\begin{abstract}
a Max Planck Institute for Human Development, Berlin, Germany
${ }^{\mathrm{b}}$ Department of Psychology, University of Amsterdam, Amsterdam, The Netherlands

${ }^{c}$ Educational Studies \& Learn!, Free University, Amsterdam, The Netherlands

d Centre for Urban Mental Health, Amsterdam, The Netherlands
\end{abstract}

\section{Acknowledgments/Funding}

MKD is supported by a Rubicon fellowship of the Netherlands Organisation for Scientific Research (NWO) no.019.191SG.005. SE is supported by a NWO VENI Grant no.016-195261. MSMS is supported by Yield Graduate Program with project 022.006.0, which is financed by NWO.

\section{Conflict of Interest}

The authors do not have any conflict of interest to declare.

\footnotetext{
${ }^{1}$ Correspondence concerning this article should be addressed to Marie K. Deserno, Max Planck Institute for Human Development, Berlin, Germany. E-mail: marie.deserno@gmail.com.
} 


\begin{abstract}
In recent years, methodological advances for analyzing developmental data are coming thick and fast. Two of the most popular and rapidly developing frameworks are (i) longitudinal structural equation modeling and (ii) network modeling. The present paper outlines the incremental gain in what we can learn from data about co-developing skills and challenges when using these two frameworks in tandem. First, we discuss the proposed analytic paradigm in the context of fundamental questions in developmental psychology. Second, we present two different paths to formalize such questions, introducing, first, a recently developed network model for longitudinal panel data and, second, the notion of growth parameter networks based on latent growth curve models. Used in tandem, they can provide new insights into the longitudinal co-development of developmental domains. Specifically, we focus on integrating growth parameters from latent growth curve models into networks and analyzing them as such. Third, we illustrate these analytic steps with an empirical example using longitudinal data from the Millenium Cohort Study ( $N=7623)$. As illustrated and discussed in the real data example, the proposed approach offers a magnifying glass to the study of coupled developmental changes. Teasing apart the processes underlying the heterogeneity of childhood development can, in turn, add to substantive developmental theory.
\end{abstract}


In developmental psychology, the increasing use of multivariate growth curve models and latent difference score models (Tucker-Drob, Brandmaier, \& Lindenberger, 2019; Kievit et al., 2018; Granic, Hollenstein \& Lichtwarck-Aschoff, 2016) attests to a growing interest in modeling developmental change beyond raw change scores. Simultaneously, a complexitybased paradigm for modeling psychological phenomena as networks has emerged and taken the field by storm (Borsboom, 2017). We here propose to integrate modeling latent change with network psychometrics to advance the study of developmental change. Therefore, our aim is threefold: First, we discuss the proposed analytic paradigm embedded in recent theoretical developments around pressing questions in developmental psychology. For example, are there variances across the lifespan rates of intraindividual longitudinal changes across different cognitive abilities (integration, differentiation and dedifferentiation hypothesis; Baltes et al., 1980; Shing, Lindenberger, Diamond, Li, \& Davidson, 2010)? And are patterns of coupled change predictive of developmental outcome, for both typically and atypically developing children? Second, we will present two different paths of tackling such questions, introducing, first, a recently developed network model for longitudinal panel data and, second, the notion of growth parameter networks based on latent growth curve models, which in tandem can provide new insights into the longitudinal co-development of developmental domains. Third, we will present an illustratory example of the aforementioned analytic paradigm using longitudinal data from the Millenium Cohort Study (MCS; e.g., Fitzsimons, 2017). More specifically, we apply the presented methods to investigate the longitudinal co-development of behavioral and emotional problems of children (age 3 to 7) using the subscales of the strengths and difficulties questionnaire (SDQ; Goodman, 1997). Also, we investigate whether the longitudinal co-developmental patterns differ for typical and atypical development as indicated by the age of 14 . 


\section{Developmental change across the lifespan}

Developmental psychology, as the discipline's name suggests, evolves around questions of developmental change: its central concept is that throughout the lifespan we observe change in multiple architectures relevant to human development, e.g. change in cognition, change in behavior or change in underlying developmental mechanisms. The integration-differentiation hypothesis, for example, proposes that intellectual abilities become integrated from infancy into childhood and differentiate again from a general ability into more specific abilities from childhood into adulthood. It proposes that accumulating influence from environmental, motivational and interest factors on intellectual development in childhood and adolescence result in growth trajectories of fluid and crystalized intelligence that become more independent (Baltes et al., 1980; Rinaldi \& Karmiloff-Smith, 2017). However, age differentiation effects in intelligence are not consistently found, potentially due to measurement invariance (Reinart, 1970). In recent efforts to accommodate the complex and contradictory empirical findings in the intelligence realm, models based on complex system approaches were put forward. The mutualism model of intelligence (van der Maas et al., 2006), for example, explains the integration-differentiation process by a complex dynamic network of interactions between individual abilities, instead of external influences. Moreover, the model predicts that the differentiation process occurs at a later age for low-intelligent groups. For executive functions, there is some evidence for the age differentiation process as well. Typically, latent factor models show that in early childhood working memory and inhibition are not separable (Wiebe, Espy, \& Charak, 2008; Wiebe et al., 2011) whereas in later childhood and adolescence these abilities are best described by multiple latent factors (Brydges, Fox, Reid, \& Anderson, 2014; Huizinga, Dolan, \& Van der Molen, 2006). This raises the question of whether the growth processes of these abilities decouple during normal 
development. In a clinical context, it has been proposed that comorbidity in early childhood is explained by the differentiation of symptoms between disorders with increasing age (Sterba et al., 2010). This also implies that the correlation patterns of symptoms and developmental challenges would change with age. These theoretical perspectives raise questions focused on whether patterns of coupled change are predictive of developmental outcome, for both typically and atypically developing children.

Although such questions are characterized by a fundamental interest in multivariate interrelations and temporal dynamics, much of the empirical literature treats the complexity of developmental data as a nuisance. The call for more complex systems thinking in developmental psychology, however, is by no means new (Van der Maas \& Molenaar, 1992; Raijmakers, Van Koten, \& Molenaar, 1996; Van Geert, 2011). In the past decades, multiple researchers have suggested that dynamical system methods can better capture, and statistically explain, developmental phenomena such as equilibration (e.g. refining mental structures; Piaget, 1978), self-organization (in the domain of learning; Köhler, 1920), or emergence (in the domain of higher-order phenomena such as intelligence; Van der Maas et al., 2006; Anderson et al., 2008). The synergy of recently developed sophisticated models of change on the one hand, and even more recent advances in network psychometrics on the other, offers a new take on fundamental questions in the field: What drives developmental change? How do developmental domains interact and do their patterns of interaction tell us anything about developmental challenges before they reinforce over time?

One could hypothesize, for example, that different developmental domains reveal different patterns of organization in specific developmental stages. Sensitive developmental stages could then be characterized by an increase in domain-specific (or local) network reactivity to influences from other domains which could be signaled by looking at such patterns. It would, therefore, be insightful to investigate the effect of change patterns of 
behavioral parameters at different developmental stages on the change patterns of another developmental domain. The behavior of a system right before a tipping point, for instance, has recently gained attention in the context of psychopathological systems that approach a point of change, e.g. from 'healthy' to 'depressed' (Wichers, Wigman \& Myin-Germeys, 2015). While on a substantively different timescale, this idea is much in line with studies of developmental phase-specific interactions between intra-individual disturbances and development (Johnson et al., 2017). Studying developmental change within a network framework, therefore, also enables us to study clinically relevant concepts, such as resilience in the face of challenges to typical functioning, on a more detailed micro-level of the system. Generally, conceptualizing developmental outcome as the result of a network of many small phase-specific interactions across domains (Van der Maas et al., 2006), and modeling individual differences in change networks will allow us to study another layer of heterogeneity in developmental trajectories.

Also, given the rapid increase of large, longitudinal cohorts (see for example this curated list of open access psychological datasets; doi10.17605/osf.io/th8ew), developmental science should be invested to get more value out of the rich existing data sources in the field to contribute to a better understanding of the mechanisms behind developmental trajectories. Simultaneously, advances in modelling development enable us to ask a novel set of questions about how developmental domains interact over time. Inevitably, every statistical model implies a set of strong assumptions about the origin and relationships among the variables under investigation. Certain kinds of information, such as the interaction between cognitive domains in atypical development, can be entirely obscured when choosing a different model. We propose the combined use of structural equation modelling and the network analytic framework to fit latent change score / latent growth curve models to longitudinal assessments of developing domains so result in so-called growth parameter networks. This statistical 
framework will provide insights into the complex interactions underlying individual differences in developmental trajectories.

\section{Networks of developmental dynamic parameters}

Complex system approaches offer a modern framework to accommodate heterogeneity in multivariate developmental systems. Rather than modeling univariate cause-effect relations, such as the wide-spread neuropsychological perspective of focal damage underlying atypicalities, complex system approaches model development as an evolving system where different domains interact over time (Van der Maas et al., 2006; Kievit et al., 2018). From this theoretical stance, the mental health realm has witnessed the emergence of a new paradigm succeeding to translate the theoretical starting point of complexity into a toolbox of network analytic tools particularly aimed at psychological data (Borsboom et al., 2017; Cramer et al., 2010). In the past decade, these tools have been applied to a variety of mental health related questions, such as the transition into mood disorders (Van de Leemput et al., 2014), comorbidity (Cramer et al., 2010; Blanken et al., 2018), vulnerability (Van Borkulo et al., 2015; Schweren et al., 2018), and the general exploration of symptom-to-symptom relations in a broad range of mental health conditions (see Fried et al., 2017 for an overview). Generally speaking, in a network model, variables are represented by nodes that form a network by their interrelations through edges, often weighted by some statistic (e.g., magnitude of partial correlation, regression coefficients). For cross sectional data used to explore dominant inter-person structures of the mental health systems, sparse partial correlation networks - also termed Gaussian graphical models (GGM) - have been put forward in various forms and for various types of variables (Lauritzen, 1996; Epskamp et al., 2018). For intraperson fluctuations, often distilled from continuous time series data, network researchers have developed a toolbox of gaussian graphical models (GGM; Epskamp et al., 2018) and vector autoregressive modeling (VAR) approaches (e.g., Bringmann et al., 2013; Haslbeck et al., 
2020), resulting in different layers of information aiming to separate relatively stable betweenperson structures from within-person dynamics (Epskamp et al., 2018). The latter type of models used to model continuous data resemble the directed structures often targeted by structural equation models (SEM).

For intra-person fluctuations, often distilled from continuous time series data, network researchers primarily rely on vector autoregressive modeling (VAR) approaches. Combining the GGM with VAR approaches has lead to the graphical VAR (GVAR; Epskamp et al., 2018) model, which is now often used in clinical psychology. Estimated from longitudinal data of multiple subjects, the GVAR model can be used to gain insights in three different levels of analysis: temporal within-person dynamics (how well do within-person fluctuations predict other within-person fluctuations over time?), contemporaneous within-person dynamics (how well do within-person fluctuations predict other within-person fluctuations in the same time-window, after controlling for temporal effects?), and between-person relations (how do stable averages relate to one-another?). In this paper, we propose two methods for incorporating GGM models for developmental data: a method based on cross-lagged panel data models, and a method based on latent growth curve models. In both methods, we can use multi-group estimation to test for differences between groups. All methods have been implemented in the open-source software package psychonetrics (Epskamp, 2020), which allows for incorporating GGM structures in general SEM.

\section{Panel GVAR models}

In recent work, the GVAR model was extended to be estimated from panel data designs (Epskamp, 2020). This model can be termed the panelGVAR model, and is structurally very similar to a stationary random-intercept cross-lagged panel model (Hamaker, Kuiper \& Grasman, 2015) with three exceptions. First, the covariance structure of innovation terms can 
be modeled as a GGM rather than as a marginal variance-covariance matrix as typical in SEM. This allows for estimating a contemporaneous network structure, informing us on what covaries within a single assessment point, not only across time. Second, the variancecovariance matrix of random intercepts can also be modeled as a GGM rather than a marginal variance-covariance matrix, which allows for estimating a between-persons network. Finally, the first wave of measurements is not treated as exogenous with a unique set of variances and covariances. Rather, a Kronecker product structure (Hamilton, 1994) is used to compute the implied variance-covariance structure of the first wave of measurement given the temporal and contemporaneous network structures as well as the assumption of stationarity. This leads to a more parsimonious model that will fit poorly if stationarity is not attained in the dataset.

As we wish to apply the panelGVAR model to developmental data, stationarity is a problem as we can naturally expect mean levels and variances to change over time. To this end, we first standardize the entire dataset, enforcing similarity in means and variances across waves. This way, we can investigate within-person and between-person relationships between the variables of interest after taking growth processes into account. We term this the partialdevelopmental panelGVAR model. Fitting this model, we can first fit a saturated model in which all edges are included. If this saturated model fits well, we can conclude that after adjusting for changes in means and variances, a lag-1 process explains the data adequately and relationships between variables are stationary over time. Next, we can remove edges (prune) that are not significant and re-estimate the model. If this sparser network fits well, we can conclude that sparse interactions can adequately explain the data.

\section{[----- INSERT FIGURE 1 ABOUT HERE ----]}

Figure 1. Formal depiction of a bivariate panelGVAR model. Here, squares represent two standardized observed variables $(\mathrm{z})$ at three different timepoints $(\mathrm{t})$ with an underlying latent temporal structure depicted

by $\mu$. 


\section{Latent growth curve models}

The second class of models we are looking to leverage in the proposed framework are latent growth curve models (LGC). These models are more and more popular to model change in mean levels of variables over time (for an extensive introduction and tutorial see Kievit et al., 2018). While often used in univariate models (only modeling growth in one variable over time), these models can also be used in a multivariate setting. In this setting, an intercept and growth latent variable are included for every variable. We can model the growth to be nonlinear with particular constrains on the factor loadings on the slope latent variables (e.g., first factor loading fixed to 0 , second fixed to 1 , and all other factor loadings free to estimate). The intercept and slope variables can be predicted in SEM using exogenous covariates, such as gender. In addition, these intercept and slopes variables also feature covariances. We propose to substantively interpret these covariance structures from a network perspective. That is, we propose to display and interpret the correlational structure of intercept and slope variables as a correlation network (Epskamp et al., 2012), or to display the partial correlational structure of intercept and slope variables in a Gaussian Graphical model (GGM). We term this framework the Latent Growth Gaussian Graphical model (LGGGM), and showcase its potential below in an example.

\section{[---- INSERT FIGURE 2 ABOUT HERE ----]}

Figure 2. Formal depiction of a bivariate latent growth curve model with freely estimated slope factor loadings at timepoint $3(\mathrm{t}=3)$. Again, squares represent observed variables and circles latent growth parameters, such as intercepts and slopes. Note that factor loadings of the slope factors to the first observations are fixed to zero (not depicted), factor loadings to the second observation re fixed to 1 , and factor loadings to the third observations are freely estimate. 


\section{Illustratory example with the longitudinal Millenium Cohort Study}

We used data from the Millennium Cohort Study (MCS; www.cls.ioe.ac.uk/mcs), a longitudinal survey which started in September of 2000 and followed a sample of individuals selected from all births in the UK within that year. The first measurement occasion took place when children were around 9 months old. The following assessment waves were conducted bi-annually from age 3 on, with sweeps 2, 3, and 4 including longitudinal measurements of the Strength and Difficulties Questionnaire (SDQ; Goodman, 1997). The SDQ is a screening instrument which is used to measure psychological problems (emotional and behavioral) and skills in children and adolescents. It includes five domains: emotional symptoms, peer problems, conduct problems, hyperactivity/inattention and prosocial behavior. Longitudinal research using the SDQ is multifaceted: previous studies have, for example, investigated the behavioral, emotional and social development within specific subgroups of children (St Clair et al., 2011; Hoekstra et al., 2013); other studies have looked at the effect of environmental risk factors (e.g., low neighborhood human capital, family socio-economic disadvantage; Flouri \& Sarmadi, 2016; Flouri, Midouhas \& Joshi, 2014; Midouhas et al., 2014) on children's development. Moreover, studies have shown that the SDQ can help detect an increase of psychopathology risk in children and adolescents (Becker et al., 2015) and can predict ADHD at school age (Rimvall et al., 2014). While there are some examples of longitudinal research using the SDQ sum score, we are not aware of any study looking at whether patterns of coupled change in the SDQ domains are predictive of outcome. We here focus on how the five domains interact and reciprocally reinforce one another over time - in other words, how change in one domain relates to change in another domain. 
Previous research has indicated reciprocal linkage of (some of) the domains included in the SDQ: A one-year longitudinal study with four waves including both children and adolescents showed that emotional problems (as measured by the SDQ) and ADHD symptoms of inattention and hyperactivity/impulsivity are linked (Han et al., 2020). In their study, Han and colleagues (2020) found that inattention symptoms were transactionally and dynamically related to emotional problems, whereas hyperactivity/impulsivity and emotional problems were related, but not reciprocally. This was tested with two separate cross-lagged panels though, thus not taking account the complex relationship between (symptom) domains. Also, externalizing and internalizing problems are known to be related to hyperactivity/impulsivity: Based on their systematic review on preschool age children, Huber, Plötner and Schmitz (2018) suggest that externalizing symptoms co-occur with deficits in prosocial behavior, whereas internalizing symptoms may coincide with either deficient or exaggerated levels of prosocial behavior.

Given that (i) scores on the SDQ have been shown to be predictive of an increased risk in psychopathology of children and adolescents (Becker et al., 2015) and (ii) studies highlighting the (reciprocal/causal) relations between dimensions of the SDQ (e.g., Han et al., 2020), we will be applying the aforementioned statistical framework to longitudinal assessments of the SDQ dimensions (3- to 7-year-olds; 3 waves) of two groups: normally developing children $(\mathrm{N}=7333)$ and children who have long lasting (12 months or more) social/ behavioral problems, such as ADHD, autism or Asperger's Syndrome at age 14 $(\mathrm{N}=290)$. As this analysis primarily serves as an illustratory example of the proposed networks of developmental dynamic parameters, we refrain from any confirmatory testing. Generally speaking, we expect positive (i.e., reinforcing) relationships between SDQ dimensions, with the exception of prosocial behavior, which is expected to be negatively related with the other dimensions, especially with externalizing problems (Huber et al., 2019). Moreover, differences 
may exist in the strength of relations between dimensions between the typical and the atypical development group.

\section{Sample}

Of the initial 18,552 families ( $N=18,818$; including twins and triplets) 11,714 participants remained in wave 6 , which we used to determine whether children's development was typical or atypical. Complete data of the two groups we were interested in existed for a sample of $\mathrm{N}$ $=7623$ (3803 girls, 3820 boys): The typical development group was made up of individuals who did not have long lasting physical or mental health conditions or illnesses ( $N=7333$; 3725 girls), whereas the atypical development group was made up of individuals who had long lasting social/ behavioral problems (i.e., ADHD, autism, Asperger's Syndrome; N = 290; 78 girls). See Table 1 for descriptive statitistics for each outcome group and assessment wave (T2-T4).

Table 1. Descriptive statistics for the SDQ dimensions per group and assessment wave.

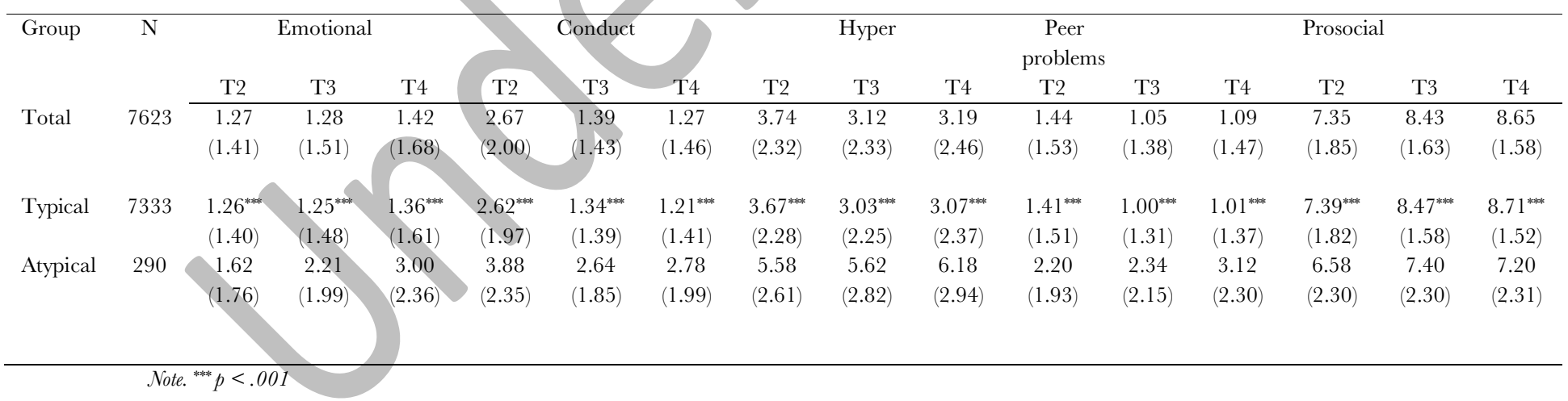

\section{Modeling framework}

We first model the temporal dynamics of standardized z-scores of the five domains of the SDQ with a graphical vector-autoregression (GVAR) model using the R-package psychonetrics version 0.7.2. (Epskamp, 2020) in R version 4.0.2. ('Taking Off Again'). Fitting the panelGVAR model allows for the same fixed-effects decomposition as known from intensive 
time-series network modeling: a temporal network, a contemporaneous network, and a between-subjects network. In a second step, we then modeled the trajectories of the SDQ domains using multivariate multi-group growth curve models. Here, we modeled the latent structure as a Gaussian Graphical Model (GGM) and estimated all residual structures using a Cholesky decomposition. In our models, we chose to group the children based on whether or not their primary care giver had indicated long-lasting behavioral problems, such as autism or ADHD at the age of 14 . We fit a range of multi-group growth curve models to test for group differences in growth parameters between the atypical group and the typically developing group. To assess model fit we inspected the Akaike Information Criterion (AIC) and the Bayesian Information Criterion (BIC). Since these analyses serve an illustratory purpose we simply note that we expect poorer absolute model fit given our small atypical group $(N=290$; DeRoche, 2009).

\section{Results}

Temporal dynamics. Figure 3 shows the estimated cross-lagged panel networks depicting the interrelations between emotional problems, peer problems, pro social activities, conduct problems and hyperactivity/inattention problems, (i) across time, (ii) within measurement occasions, and (iii) between-subject variations.

\section{[---- INSERT FIGURE 3 ABOUT HERE ----]}

Figure 3. Estimated directed temporal network, estimated contemporaneous partial correlation network and the estimated between-subjects partial correlation network. Blue edges represent positive (enhancing) relationships within or across time while red edges represent negative relationships. Abbreviations are: peerp - problems with peers. presoc - pro-social behavior. condpr - conduct problems. hyper hyperactivity/inattention. emo - emotional problems.

The temporal network suggests that hyperactivity problems self-reinforce over time (depicted by the blue arrow going from the variable to itself) and drive (later) peer problems 
and emotional problems as well as predicting reduced prosocial behavior. Conduct problems and hyperactivity show a reciprocal reinforcing relationship, whereas conduct problems are negatively reciprocally linked with prosocial behavior. The contemporaneous network adds information about covariation within assessment points, suggesting that hyperactivity/inattention problems and conduct problems go hand-in-hand, and that hyperactivity/inattention problems co-occur with emotional problems and reduced prosocial behavior. The between-subjects network highlights the strong relations between hyperactivity and conduct problems once again; it also shows that hyperactivity/inattention problems are related with peer problems and with both reduced emotional problems and reduced prosocial behavior. Comparing the contemporaneous and the between-subjects network, the relation between hyperactivity/inattention symptoms and emotional problems stands out: Whereas the relationship between the two constructs is positive in the contemporaneous network, indicating co-occurrence at the same time point, it is negative in the between-subjects network, revealing that across children, if one child has high levels of hyperactivity/inattention symptoms, they have lower levels of emotional problems within that same measurement occasion.

Group differences in growth parameter networks. In a second step, we tested for differences and similarities between children that develop behavioral problems $(\mathrm{N}=290)$ and those that develop typically $(\mathrm{N}=7333)$ by adopting multi-group LGMs. In the model comparisons, we tested for group differences in specific parameters while constraining all other parameters in the model to be equal across the two groups. We start out with a model specifying networks to be equal, followed by a model with equal growth between the groups, followed by a model with both equal networks and equal growth, followed by a model with equal factor loadings in addition to equal networks and equal growth. This succession of model fits is shown in Table 2. 
Table 2. Model fit indices for different multigroup models based on the five SDQ subdomains with freed model parameters as indicated in the first column (Parameters).

\begin{tabular}{|c|c|c|c|c|c|c|c|}
\hline Parameters & df & $\mathbf{X}^{2}$ & $\Delta \mathbf{X}^{2 \diamond}$ & $\boldsymbol{p}$ & AIG & BIC & RMSEA \\
\hline $\begin{array}{c}\text { Equal } \\
\text { networks } \\
\text { (omega) }\end{array}$ & 165 & 2348.93 & 151.10 & $<0.0001$ & 276898.81 & 277627.39 & 0.059 \\
\hline $\begin{array}{l}\text { Equal means } \\
\text { (eta) }\end{array}$ & 130 & 2486.03 & 256.81 & $<0.0001$ & 277105.91 & 278077.36 & 0.069 \\
\hline $\begin{array}{c}\text { Equal } \\
\text { networks }+ \\
\text { equal means }\end{array}$ & 175 & 2762.94 & 389.88 & $<0.0001$ & 277292.82 & 277952.02 & 0.062 \\
\hline $\begin{array}{l}\text { Equal growth } \\
\text { (lambda) }\end{array}$ & 125 & 2229.22 & 28.82 & $<0.0001$ & 276859.10 & 277865.24 & 0.066 \\
\hline $\begin{array}{c}\text { Equal } \\
\text { networks }+ \\
\text { equal growth }\end{array}$ & 170 & 2373.07 & 24.14 & $<0.001$ & 276912.95 & $277606.84^{*}$ & 0.058 \\
\hline All different & 120 & 2200.40 & & & $276840.28 *$ & 277881.12 & 0.067 \\
\hline
\end{tabular}

In short, based on the BIG the model fit suggests the model with equal networks and equal growth to be preferred. Although the AIC suggests that the model allowing for group differences in all parameters shows the best fit, we here only discuss the growth parameter network for the full sample. In case of conclusive evidence for group difference in the partial correlation matrices of the groups, a different network for each group can be extracted. In our empirical example, however, group sizes were very different $(N=7333$ vs. $N=290)$ which will impact the comparative fit statistics. We show the different networks for the two groups in Figure 4, but refrain from further analysis of these networks as the network of the group with behavioral problems (b) is clearly underpowered (the dashed network edges indicate nonsignificance). If one were to find meaningful group differences, the growth parameters networks can be further analyzed through network analytic tools. Group differences could 
manifest in the e.g., density of connections, in the strength of connections or in the role specific nodes play in the network.

Simultaneously, the growth parameter network offers a broad range of insights into specific temporal interrelations between problem domains. The full sample growth parameter network in Figure 4 (c), for example, suggests that more initial hyperactivity/inattention problems (int_hyper) drive a steeper increase in the conduct problem domain (slope_condpr), as well as in the peer problem domain (slope_peerpr). Initial levels of hyperactivity/inattention problems co-occur with initial levels of conduct (int_condpr) and peer problems (int_peerpr). The increase of hyperactivity/inattention problems (slope_hyper) from age 3 to 7 is related to increasing emotional problems (slope_emo) and a decrease of both peer problems (slope_peerpr) and prosocial behavior (slope_prosoc). High initial levels of emotional problems (int_emo) do not necessarily go hand-in-hand with the growth of hyperactivity/inattention problems. Finally, the parameters of conduct problems and prosocial behavior are negatively related: If the initial starting value of one of the two constructs is high, the other one's initial levels are low; high initial levels on one of the two are related with reduced growth of the other; and lastly, growth on one of the two is associated with decrease in the other construct.

As mentioned earlier, certain patterns of relations between growth parameters which may indicate processes of differentiation and integration, are of especial interest for developmental psychology. Relations of growth parameters that may signify differentiation of constructs across time are high partial correlations of initial levels of two variables, coupled with low (or non-existing) partial correlations of the change of the same two variables across time. Such a pattern exists, for instance for the hyperactivity/inattention and conduct problems: While they are initially highly correlated - indicating that they co-occur in threeyear-old's, their slopes are not related - change in one of the two constructs is independent 
from change in the other construct. Relations of growth parameters which may signify integration of two constructs across time are reversed: Initially, the two constructs are not related, whereas their growth is highly correlated. Initial levels of emotional and peer problems, for instance, are not significantly correlated, whereas their slopes are; an increase of emotional problems from the age of 3 to 7 thus co-occurs with an increase of peer problems.

Moreover, the negative relationship between initial levels of a construct and its growth may indicate (1) either that a ceiling effect exists in the construct, i.e. that if a child starts out relatively high on a problem domain there is only so much left to grow even more problems in that regard; or that (2) the sensitive period in which a problem domain developed has already passed, i.e. that there is variance in the timing of growth processes. Ultimately, this may signify that there are differences in the process of change across constructs: Whereas some SDQ domain scores are high in early age (such as conduct problems, having a negative relation between intercept and slope), others may be higher later on (such as hyperactivity/inattention problems, with no relation between intercept and slope).

[---- INSERT FIGURE 4 ABOUT HERE ----]

Figure 4. Growth parameter networks for (a) the group of children without behavioral problems at age 14, (b) the group with behavioral problems at age 14, and (c) the full sample $(\mathrm{N}=7623)$. Blue edges represent positive(enhancing) relationships within or across time while red edges represent negative relationships. Dashed lines indicate non-significant relationships. Abbreviations are a combination of int for intercept and slope for slope parameters with an abbreviation for the SDQ domain: peerp - problems with peers. presoc - pro-social behavior. condpr - conduct problems. hyper - hyperactivity/inattention. emo emotional problems.

Temporal dynamics and growth parameter networks. The results given the (change of) mean differences on the SDQ domains across time and individuals, as shown in the temporal dynamics networks, and the interaction of initial levels and change across SDQ domains, as shown in the growth parameter networks, can be used to draw a more complete 
picture of the complex longitudinal dynamic interaction of domains. While the temporal network shows a reciprocal positive relation between hyperactivity/inattention and conduct problems, for instance (see Figure 3a), the relationship of initial levels on both domains and their growth parameters paints a more complex picture: Initial levels of hyperactivity/inattention problems are related with both initial levels of conduct problems as well as increase of conduct problems over time (see Figure 4c). Increases of hyperactivity/inattention problems over the years are not explained by any of the growth parameters of conduct problems, though; highlighting that to explain growth in hyperactivity/inattention problems, other domains have to be considered. The relationship between prosocial behavior and conduct problems, on the other hand, is more straightforward: The two constructs are shown to be negatively reciprocally linked in the temporal network (see Figure 3a), which is also the case in the growth network. Initial levels in prosocial behavior (int_prosoc) and conduct problems (int_condpr) are negatively related, as are the initial levels of conduct problems with the change in prosocial behavior (slope_prosoc) and vice versa - when exhibiting conduct problems early on, children are less likely to increase their prosocial behavior - and the two growth parameters, meaning that if a child increases their prosocial behavior, their conduct problems decrease, and vice versa.

\section{Conclusion}

In this contribution, we propose a combined framework of longitudinal SEM with network modeling to be a valuable addition to the methodological toolbox for developmental questions in child and adolescent psychology. In particular, we propose the use of latent growth curve models and panel graphical models in tandem. In doing so, developmental researchers can combine their interest in coupled cross-domain growth and coupled crossdomain means of developing skills and challenges in an intuitive network framework. As our 
illustratory example using existing longitudinal data from the Millenium Cohort Study shows, the proposed framework offers insights in reciprocal relations between developmental processes as well as group differences within such processes, which are not easily available using other modeling methods. We believe that the proposed framework may, therefore, aid in tackling fundamental developmental questions rooted in developmental psychology. 


\section{References}

Anderson, G. M. (2008). The potential role for emergence in autism. Autism Research, 1(1), 1830.

Baltes, P.B., Cornelius, S.W., Spiro, A., Nesselroade, J.R. \& Willis, S.L. (1980) Integration versus differentiation of fluid/crystallized intelligence in old age. Developmental Psychology. 16, $625-635$.

Becker, A., Rothenberger, A., Sohn, A., \& BELLA Study Group. (2015). Six years ahead: a longitudinal analysis regarding course and predictive value of the Strengths and Difficulties Questionnaire (SDQ) in children and adolescents. European Child \& Adolescent Psychiatry, 24(6), $715-725$.

Blanken, T. F., Deserno, M. K., Dalege, J., Borsboom, D., Blanken, P., Kerkhof, G. A., \& Cramer, A. O. (2018). The role of stabilizing and communicating symptoms given overlapping communities in psychopathology networks. Scientific Reports, 8(1), 1-8.

Borsboom, D. (2017). A network theory of mental disorders. World Psychiatry, 16(1), 5-13.

Bringmann, L. F., Vissers, N., Wichers, M., Geschwind, N., Kuppens, P., Peeters, F., ... \& Tuerlinckx, F. (2013). A network approach to psychopathology: new insights into clinical longitudinal data. PloS one, 8(4), e60188.

Brydges, G. R., Fox, A. M., Reid, C. L., \& Anderson, M. (2014). The differentiation of executive functions in middle and late childhood: A longitudinal latent-variable analysis. Intelligence, 47, 34-43.

Cramer, A. O., Waldorp, L. J., Van Der Maas, H. L., \& Borsboom, D. (2010). Comorbidity: A network perspective. Behavioral and Brain Sciences, 33(2-3), 137.

DeRoche, K. K. (2009). Functioning of global fit statistics in latent growth curve modeling. Unpublished Doctoral Thesis. 
Epskamp, S., Cramer, A. O., Waldorp, L. J., Schmittmann, V. D., \& Borsboom, D. (2012). qgraph: Network visualizations of relationships in psychometric data. Journal of Statistical Software, 48(4), 1-18.

Epskamp, S., Waldorp, L. J., Mõttus, R., \& Borsboom, D. (2018). The Gaussian graphical model in cross-sectional and time-series data. Multivariate Behavioral Research, 53(4), 453-480.

Epskamp, S., van Borkulo, C. D., van der Veen, D. C., Servaas, M. N., Isvoranu, A. M., Riese, H., \& Cramer, A. O. (2018). Personalized network modeling in psychopathology: The importance of contemporaneous and temporal connections. Clinical Psychological Science, 6(3), 416-427.

Epskamp, S. (2020). psychonetrics: Structural Equation Modeling and Confirmatory Network Analysis. R package version 0.7.5. https://github.com/SachaEpskamp/psychonetrics

Fitzsimons, E. (2017). Millennium Cohort Study. Sixth Survey 2015-2016. User Guide.

Flouri, E., Midouhas, E., \& Joshi, H. (2014). Family poverty and trajectories of children's emotional and behavioural problems: the moderating roles of self-regulation and verbal cognitive ability. Fournal of Abnormal Child Psychology, 42(6), 1043-1056.

Flouri, E., \& Sarmadi, Z. (2016). Prosocial behavior and childhood trajectories of internalizing and externalizing problems: The role of neighborhood and school contexts. Developmental Psychology, 52(2), 253.

Fried, E. I., van Borkulo, C. D., Cramer, A. O., Boschloo, L., Schoevers, R. A., \& Borsboom, D. (2017). Mental disorders as networks of problems: a review of recent insights. Social Psychiatry and Psychiatric Epidemiology, 52(1), 1-10.

Goodman, R. (1997). The Strengths and Difficulties Questionnaire: a research note. Fournal of Child Psychology and Psychiatry, 38(5), 581-586. 
Goodman, R., Ford, T., Simmons, H., Gatward, R., \& Meltzer, H. (2000). Using the Strengths and Difficulties Questionnaire (SDQ) to screen for child psychiatric disorders in a community sample. The British Fournal of Psychiatry, 177(6), 534-539.

Granic, I., Hollenstein, T., \& Lichtwarck-Aschoff, A. (2016). A survey of dynamic systems methods for developmental psychopathology. Developmental psychopathology, 1-43.

Hamaker, E. L., Kuiper, R. M., \& Grasman, R. P. (2015). A critique of the cross-lagged panel model. Psychological Methods, 20(1), 102.

Hamilton, J. D. (1994). Time series analysis. Princeton, NJ: PrincetonUniversity Press.

Han, G. T., Chen, Y. L., Tsai, F. J., \& Gau, S. S. F. (2020). Temporal and reciprocal relations between ADHD symptoms and emotional problems in school-age children. Fournal of Attention Disorders, 24(7), 1032-1044.

Haslbeck, J. M., Bringmann, L. F., \& Waldorp, L. J. (2020). A Tutorial on Estimating TimeVarying Vector Autoregressive Models. Multivariate Behavioral Research, 1-30.

Hoekstra, P. J., Lundervold, A.J., Lie, S. A., Gillberg, C., \& Plessen, K. J. (2013). Emotional development in children with tics: a longitudinal population-based study. European child \& adolescent psychiatry, 22(3), 185-192.

Huber, L., Plötner, M., \& Schmitz, J. (2019). Social competence and psychopathology in early childhood: a systematic review. European Child \& Adolescent Psychiatry, 28(4), 443-459.

Huizinga, M., Dolan, C. V., \& Van der Molen, M. W. (2006). Age-related change in executive function: Developmental trends and a latent variable analysis. Neuropsychologia, 44(11), 2017-2036.

Johnson, M. H. (2017). Autism as an adaptive common variant pathway for human brain development. Developmental Cognitive Neuroscience, 25, 5-11. 
Kievit, R. A., Brandmaier, A. M., Ziegler, G., Van Harmelen, A. L., de Mooij, S. M., Moutoussis, M., ... \& Lindenberger, U. (2018). Developmental cognitive neuroscience using latent change score models: A tutorial and applications. Developmental Cognitive Neuroscience, 33, 99-117.

Köhler, W. Die physischen Gestalten in Ruhe und im stationären Zustand. Vieweg, Braunschweig, 1920.

Lauritzen, S. L. (1996). Graphical models (Vol. 17). Clarendon Press.

Midouhas, E., Kuang, Y., \& Flouri, E. (2014). Neighbourhood human capital and the development of children' s emotional and behavioural problems: The mediating role of parenting and schools. Health \& Place, 27, 155-161.

Piaget, J. (1978). La formation du symbole chez l'enfant: imitation, jeu et rêve, image et représentation. FeniXX.

Raijmakers, M. E., Van Koten, S., \& Molenaar, P. C. (1996). On the validity of simulating stagewise development by means of PDP networks: Application of catastrophe analysis and an experimental test of rule-like network performance. Cognitive Science, 20(1), 101-136.

Reinert, G. (1970). Comparative factor analytic studies of intelligence throughout the human life-span. In L. R. Goulet \& P. B. Baltes (Eds.), Life-span developmental psychology: Research and theory. New York: Academic Press.

Rimvall, M. K., Elberling, H., Rask, C. U., Helenius, D., Skovgaard, A. M., \& Jeppesen, P. (2014). Predicting ADHD in school age when using the Strengths and Difficulties Questionnaire in preschool age: a longitudinal general population study, GCC2000. European Child E Adolescent Psychiatry, 23(11), 1051-1060.

Rinaldi, L., \& Karmiloff-Smith, A. (2017). Intelligence as a developing function: A neuroconstructivist approach. Fournal of Intelligence, 5(2), 18. 
Schweren, L., van Borkulo, C. D., Fried, E., \& Goodyer, I. M. (2018). Assessment of symptom network density as a prognostic marker of treatment response in adolescent depression. FAMA Psychiatry, 75(1), 98-100.

Shing, Y. L., Lindenberger, U., Diamond, A., Li, S. C., \& Davidson, M. C. (2010). Memory maintenance and inhibitory control differentiate from early childhood to adolescence. Developmental Neuropsychology, 35(6), 679-697.

St Clair, M. C., Pickles, A., Durkin, K., \& Conti-Ramsden, G. (2011). A longitudinal study of behavioral, emotional and social difficulties in individuals with a history of specific language impairment (SLI). Fournal of Communication Disorders, 44(2), 186-199.

Sterba, S. K., Copeland, W., Egger, H. L., Jane Costello, E., Erkanli, A., \& Angold, A. (2010). Longitudinal dimensionality of adolescent psychopathology: Testing the differentiation hypothesis. Fournal of Child Psychology and Psychiatry, 51(8), 871-884.

Tucker-Drob, E. M., Brandmaier, A. M., \& Lindenberger, U. (2019). Coupled cognitive changes in adulthood: A meta-analysis. Psychological Bulletin, 145(3), 273.

Van Borkulo, C., Boschloo, L., Borsboom, D., Penninx, B. W., Waldorp, L. J., \& Schoevers, R. A. (2015). Association of symptom network structure with the course of depression. JAMA Psychiatry, 72(12), 1219-1226.

Van de Leemput, I. A., Wichers, M., Cramer, A. O., Borsboom, D., Tuerlinckx, F., Kuppens, P., ... \& Derom, C. (2014). Critical slowing down as early warning for the onset and termination of depression. Proceedings of the National Academy of Sciences, 111(1), 87-92.

Van der Maas, H. L., \& Molenaar, P. C. (1992). Stagewise cognitive development: an application of catastrophe theory. Psychological Review, 99(3), 395.

Van Der Maas, H. L., Dolan, C. V., Grasman, R. P., Wicherts, J. M., Huizenga, H. M., \& Raijmakers, M. E. (2006). A dynamical model of general intelligence: the positive manifold of intelligence by mutualism. Psychological Review, 113(4), 842. 
Van Geert, P. (2011). The contribution of complex dynamic systems to development. Child Development Perspectives, 5(4), 273-278.

Wichers, M., Wigman, J. T. W., \& Myin-Germeys, I. (2015). Micro-level affect dynamics in psychopathology viewed from complex dynamical system theory. Emotion Review, 7(4), 362367.

Wiebe, S. A., Espy, K. A., \& Charak, D. (2008). Using confirmatory factor analysis to understand executive control in preschool children: I. Latent structure. Developmental Psychology, 44(2), 575.

Wiebe, S. A., Sheffield, T., Nelson, J. M., Clark, C. A., Chevalier, N., \& Espy, K. A. (2011). The structure of executive function in 3-year-olds. Fournal of Experimental Child Psychology, 108(3), 436-452. 